\title{
Universiteit
}

Leiden

The Netherlands

\section{Antibunched photons emitted by a quantum point contact out of equilibrium}

Beenakker, C.W.J.; Schomerus, H.

\section{Citation}

Beenakker, C. W. J., \& Schomerus, H. (2004). Antibunched photons emitted by a quantum point contact out of equilibrium. Retrieved from https://hdl.handle.net/1887/1295

Version: $\quad$ Not Applicable (or Unknown)

License: $\quad$ Leiden University Non-exclusive license

Downloaded from: https://hdl.handle.net/1887/1295

Note: To cite this publication please use the final published version (if applicable). 


\title{
Antibunched Photons Emitted by a Quantum Point Contact out of Equilibrium
}

\author{
C.W. J. Beenakker ${ }^{1}$ and H. Schomerus ${ }^{2}$ \\ 'Instituut-Lorentz, Universiteit Leiden, P.O. Box 9506, 2300 RA. Leiden, The Netherlands \\ ${ }^{2}$ Max-Planck-Institut fur Phystk komplexer Systeme, Nothnizer Strasse 38, 01187 Dresden, Germany'
}

(Received 2 May 2004; published 23 August 2004)

\begin{abstract}
Motivated by the experimental search for "GHz nonclassical light," we identify the conditions under which current fluctuations in a narrow constriction generate sub-Poissonian radiation. Antibunched electrons gencrically produce bunched photons, because the same photon mode can be populated by electrons decaying independently from a range of initial energies. Photon antibunching becomes possible at frequencies close to the applied voltage $V \times e / \hbar$, when the initial energy range of a decaying electron is restricted. The condition for photon antibunching in a narrow frequency interval below $\mathrm{eV} / \hbar$ reads $\left[\sum_{n} T_{n}\left(1-T_{n}\right)\right]^{2}<2 \sum_{n}\left[T_{n}\left(1-T_{n}\right)\right]^{2}$, with $T_{n}$ an eigenvalue of the transmission matrix. This condition is satisfied in a quantum point contact, where only a single $T_{n}$ differs from 0 or 1 . The photon statistics is then a superposition of binomial distributions.
\end{abstract}

PACS numbers: $73.50 \mathrm{Td}, 4250 \mathrm{Ar}, 42.50 \mathrm{Lc}, 73.23-\mathrm{b}$

In a recent experiment [1], Gabelli et al. have measured the deviation from Poisson statistics of photons emitted by a resistor in equilibrium at $\mathrm{mK}$ temperatures. By cross correlating the power fluctuations they detected photon bunching, meaning that the variance $\operatorname{Var} n=\left\langle n^{2}\right\rangle-\langle n\rangle^{2}$ in the number of detected photons exceeds the mean photon count $\langle n\rangle$. Their experiment is a variation on the quantum optics experiment of Hanbury Brown and Twiss [2], but now at $\mathrm{GHz}$ frequencies.

In the discussion of the implications of their novel experimental technique, Gabelli et al. noticed that a general theory [3] for the radiation produced by a conductor out of equilibrium implies that the deviation from Poisson statistics can go either way: Super-Poissonian fluctuations (Varn $\rangle\langle n\rangle$, signaling bunching) are the rule in conductors with a large number of scattering channels, while sub-Poissonian fluctuations ( $\operatorname{Var} n<\langle n\rangle$, signaling antibunching) become possible in few-channel conductors. They concluded that a quantum point contact could therefore produce $\mathrm{GHz}$ nonclassical light [4].

It is the purpose of this work to identify the conditions under which electronic shot noise in a quantum point contact can generate antibunched photons. The physical picture that emerges differs in one essential aspect from electron-hole recombination in a quantum dot or quantum well, which is a familiar source of sub-Poissonian radiation [5-7]. In those systems the radiation is produced by transitions between a few discrete levels. In a quantum point contact the transitions cover a continuous range of energies in the Fermi sea. As we will see, this continuous spectrum generically prevents antibunching, except at frequencies close to the applied voltage.

Before presenting a quantitative analysis, we first discuss the mechanism in physical terms. As depicted in Fig. 1, electrons are injected through a constriction in an energy range $e V$ above the Fermi energy $E_{F}$, leaving behind holes at the same energy. The statistics of the charge $Q$ transferred in a t1me $\tau \gg \hbar / e V$ is binomial [8], with $\operatorname{Var} Q / e<\langle Q / e\rangle$. This electron antibunching is a result of the Pauli principle. Each scattering channel $n=1,2, \ldots, N$ in the constriction and each energy interval $\delta E=\hbar / \tau$ contributes independently to the charge statistics. The photons excited by the electrons would inherit the antibunching if there would be a one-to-one correspondence between the transfer of an electron and the population of a photon mode. Generically, this is not what happens: A photon of frequency $\omega$ can be excited by each scattering channel and by a range $e V-\hbar \omega$ of initial energies. The resulting statistics of photocounts is negative-binomial [3], with $\operatorname{Var} n\rangle\langle n\rangle$. This is the same photon bunching as in black-body radiation [9].
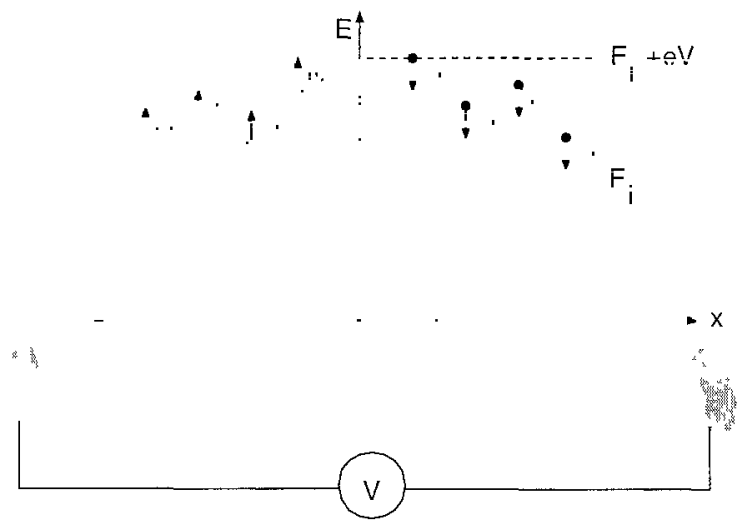

FIG. 1. Schematic diagram of a constriction in a conductor (bottom) and the energy range of electronic states (top), showing excitations of electrons (black dots) and holes (white dots) in the Fermi sea. A voltage $V$ drops over the constriction. Electrons (holes) in an energy range $e V-\hbar \omega$ can populate a photon mode of frequency $\omega$, by decaying to an empty (filled) state closer to the Fermi level 
In order to convert antibunched electrons into ant1bunched photons, it is sufficient to ensure a one-to-one correspondence between electron modes and photon modes. This can be realized by concentrating the current fluctuations in a single scattering channel and by restricting the energy range $e V-\hbar \omega$. Indeed, in a singlechannel conductor and in a narrow frequency range $\omega \leqslant$ $e V / \hbar$ we obtain sub-Poissonian photon statistics regardless of the value of the transmission probability. In the more general multichannel case, photon antibunching is found if $\left[\sum_{n} T_{n}\left(1-T_{n}\right)\right]^{2}<2 \sum_{n}\left[T_{n}\left(1-T_{n}\right)\right]^{2}$ (with $T_{n}$ an eigenvalue of the transimission matrix product $t t^{\dagger}$ )

The starting point of our quantitative analysis is the general relationship of Ref. [3] between the photocount distribution $P(n)$ and the expectation value of an ordered exponential of the electrical current operator:

$$
\begin{gathered}
P(n)=\frac{1}{n !} \lim _{\xi \rightarrow-1} \frac{d^{n}}{d \xi^{n}} F(\xi) \\
F(\xi)=\left\langle\mathcal{O} \exp \left[\xi \int_{0}^{\infty} d \omega \gamma(\omega) I^{\dagger}(\omega) I(\omega)\right]\right\rangle .
\end{gathered}
$$

We summarize the notation. The function $F(\xi)=$ $\sum_{k=0}^{\infty}\left(\xi^{k} / k^{1}\right)\left\langle n^{h}\right\rangle_{1}$ is the generating function of the factorial moments $\left\langle n^{k}\right\rangle_{1} \equiv\langle n(n-1)(n-2) \cdots(n-k+1)\rangle$. The current operator $I=I_{\text {out }}-I_{\text {mn }}$ is the difference of the outgoing current $I_{\text {out }}$ (away from the constriction) and the incoming current $I_{1 n}$ (toward the constiction). The symbol $\mathcal{O}$ indicates ordering of the curient operatots from left to right in the order $I_{1 n}^{\dagger}, I_{\text {out }}^{\dagger}, I_{\text {out }}, I_{1 n}$ The real frequency-dependent response function $\gamma(\omega)$ is proportronal to the coupling strength of conductor and photodetector and proportional to the detector efficiency. Positive (negative) $\omega$ corresponds to absorption (emission) of a photon by the detector. We consider photodetection by absor ption, hence $\gamma(\omega) \equiv 0$ for $\omega \leq 0$. Integrals over frequency should be interpreted as sums over discrete modes $\omega_{p}=p \times 2 \pi / \tau, p=1,2,3, \ldots$ The detection time $\tau$ is sent to infinity at the end of the calculation. We denote $\gamma_{p}=\gamma\left(\omega_{p}\right) \times 2 \pi / \tau$, so that $\int d \omega \gamma(\omega) \rightarrow$ $\sum_{p} \gamma_{p}$. For ease of notation we set $\hbar=1, e=1$.

The exponent in Eq. (2) is quadtatic in the current operators, which complicates the calculation of the expectation value. We remove this complication by introducing a Gaussian field $z(\omega)$ and performing a HubbardStratonovich transformation,

$$
\begin{aligned}
F(\xi)= & \left\langle\mathcal { O } \operatorname { e x p } \left[\sqrt { \xi } \int _ { 0 } ^ { \infty } d \omega \gamma ( \omega ) \left( z(\omega) I^{\dagger}(\omega)\right.\right.\right. \\
& +z(\omega) I(\omega))]\rangle
\end{aligned}
$$

The angular brackets now indicate both a quantum mechanical expectation value of the current operators and a classical average over independent complex Gaussian variables $z_{p}=z\left(\omega_{p}\right)$ with zero mean and variance $\left\langle\left|z_{p}\right|^{2}\right\rangle=1 / \gamma_{p}$.

We assume zero temperature, so that the incoming current is noiseless. We may then replace $I$ by $I_{\text {out }}$ and restrict ourselves to energies $\varepsilon$ in the range $(0, V)$ above $E_{F}$ Let $b_{n}^{\dagger}(\varepsilon)$ be the operator that creates an outgorng electron in scattering channel $n$ at energy $\varepsilon$. The outgoing current is given in terms of the electron operators by

$$
I_{\text {out }}(\omega)=\int_{0}^{V} d \varepsilon \sum_{n} b_{n}^{\dagger}(\varepsilon) b_{n}(\varepsilon+\omega)
$$

Energy $\varepsilon_{p}=p \times 2 \pi / \tau$ is discretized in the same way as frequency. The energy and channel indices $p, n$ are collected in a vector $b$ with elements $b_{p n}=$ $(2 \pi / \tau)^{1 / 2} b_{n}\left(\epsilon_{p}\right)$. Substitution of Eq. (4) into Eq (3) gives

$$
F(\xi)=\left\langle e^{b^{\dagger} Z b} e^{b^{\dagger} Z^{\dagger} b}\right\rangle
$$

The exponents contain the product of the vectors $b, b^{\dagger}$ and a matrix $Z$ with elements $Z_{p n p^{\prime} n^{\prime}}=$ $\xi^{1 / 2} \delta_{n n^{\prime}} z_{p-p^{\prime}} \gamma_{p-p^{\prime}}$. Notice that $Z$ is diagonal in the channel indices $n, n^{\prime}$ and lower-triangular in the energy ndices $p, p^{\prime}$.

Because of the ordering $\mathcal{O}$ of the current operators, the single exponential of Eq. (3) factorizes into the two noncommuting exponentials of Eq. (5). In order to evaluate the expectation value efficiently, we would like to bring this back to a single exponential - but now with normal ordering $\mathcal{N}$ of the fermion creation and annihilation operators. (Normal ordering means $b^{\dagger}$ to the left of $b$, with a minus sign for each permutation ) This is accomplished by means of the operator identity [10]

$$
\prod_{t} e^{b^{\dagger} A_{t} b}=\mathcal{N} \exp \left[b^{\dagger}\left(\prod_{i} e^{A_{\imath}-1}\right) b\right]
$$

valid for any set of matrices $A$, The quantum mechanical expectation value of a normally ordered exponential is a determinant [11],

$$
\left\langle\mathcal{N} e^{b^{\dagger} A b}\right\rangle=\operatorname{Det}(1+A B), \quad B_{\eta}=\left\langle b_{1}^{\dagger} b_{l}\right\rangle
$$

In our case $A=e^{Z} e^{Z^{\dagger}}-1$ and $B=t t^{\dagger}$, with $t$ the $N \times N$ transmission matrix of the constriction.

In the experimentally ielevant case $[1,12]$ the response function $\gamma(\omega)$ is sharply peaked at a frequency $\Omega \leq V$, with a width $\Delta \ll \Omega$. We assume that the energy dependence of the transmission matıx may be disregarded on the scale of $\Delta$, so that we may choose an $\varepsilon$-independent basis in which $t t^{\dagger}$ is diagonal. The diagonal elements are the transmission eigenvalues $T_{1}, T_{2}, \ldots T_{N} \in(0,1)$. Combining Eqs. (5) $-(7)$ we arrive at 


$$
\begin{aligned}
F(\xi) & =\left\langle\prod_{n=1}^{N} \operatorname{Det}\left[1+T_{n}\left(e^{Z} e^{Z^{\dagger}}-1\right)\right]\right\rangle \\
& =\left\langle\prod_{n=1}^{N} \operatorname{Det}\left[\left(1-T_{n}\right) e^{-Z^{\dagger}}+T_{n} e^{Z}\right]\right\rangle
\end{aligned}
$$

(In the second equality we used that Det $e^{Z^{\dagger}}=1$, since $Z$ is a lower-triangular matix) The remaining average is over the Gaussian vatiables $z_{p}$ contarned in the matı $Z$

Since the interesting new physics occuis when $\Omega$ is close to $V$, we simplify the analysis by assuming that $\gamma(\omega) \equiv 0$ for $\omega<V / 2$ Fo1 such a response function one has $Z^{2}=0$ (This amounts to the statement that no election with excitation eneigy $\varepsilon<V$ can pioduce more than a single photon of frequency $\omega>V / 2$ ) We may therefore replace $e^{Z} \rightarrow 1+Z$ and $e^{-L^{\dagger}} \rightarrow 1-Z^{\dagger}$ in Eq (8) We then apply the mati1x identity

$$
\operatorname{Det}(1+A+B)=\operatorname{Det}(1-A B) \quad \text { if } A^{2}=0=B^{2},
$$

and obtain

$F(\xi)=\prod_{p} \frac{\gamma_{p}}{\pi} \int d^{2} z_{p} e^{-\gamma_{1}, \mid \uparrow} \times \prod_{n=1}^{N} \operatorname{Det}\left[1+T_{n}\left(1-T_{n}\right) \xi X\right]$

We have defined $\xi X \equiv Z Z^{\dagger}$ and witten out the Gaussian average The Hermitian matıx $X$ has elements

$$
X_{p p}=\sum_{q} z_{p-q} z_{p^{\prime}-q} \gamma_{p-q} \gamma_{p^{\prime}-q}
$$

The integeis $p, p^{\prime}, q$ 1ange from 1 to $V \tau / 2 \pi$

The Gaussian average is easy if the dimensionless shot no1se powet $S=\sum_{n} T_{n}\left(1-T_{n}\right)$ is $\gg 1$ We may then do the integials of Eq (10) in saddle-point appioximation, with the result [13]

$$
\ln F(\xi)=-\frac{\tau}{2 \pi} \int_{0}^{V} d \omega \ln [1-\xi S \gamma(\omega)(V-\omega)]
$$

The logarithm $\ln F(\xi)$ is the generating function of the factor lal cumulants $\left\langle\left\langle n^{h}\right\rangle\right\rangle_{1}$ [14] By expanding Eq (12) in powers of $\xi$ we find

$$
\left\langle\left\langle n^{k}\right\rangle\right\rangle_{1}=(k-1)^{\prime} \frac{\tau}{2 \pi} \int_{0}^{V} d \omega[S \gamma(\omega)(V-\omega)]^{k}
$$

Equations (12) and (13) repiesent the multimode supe1position of independent negative-binomial distibutions [9] All factorial cumulants are positive, in particulat, the second, so Vai $n>\langle n\rangle$ This is supei-Poissonian radiation

When $S$ is not $\gg 1$, e g, when only a single-channel contributes to the shot noise, the iesult (12) and (13) remains valid if $V-\Omega \gg \Delta$ This was the conclusion of Ref [3], that nar1ow-band detection leads genen ically to a negative-binomial distribution However, the saddle- point appioximation bieaks down when the detection fiequency $\Omega$ appioaches the applied voltage $V$ For $V-$ $\Omega \leqq \Delta$ one has to calculate the integials in Eq (10) exactly

We have evaluated the generating function (10) for a response function of the block for $\mathrm{m}$

$$
\gamma(\omega)= \begin{cases}\gamma_{0} & \text { if } V-\Delta<\omega<V, \\ 0 & \text { if } \omega<V-\Delta,\end{cases}
$$

with $\Delta<V / 2$ The trequency dependence for $\omega>V$ is it lelevant In the case $N=1$ of a single channel, with transmission probability $T_{1} \equiv T$, we find [15]

$$
\begin{aligned}
\ln F(\xi) & =\frac{\tau}{2 \pi} \int_{V-\Delta}^{V} d \omega \ln \left[1+\xi \gamma_{0} T(1-T)(V-\omega)\right] \\
& =\frac{\tau \Delta}{2 \pi} \frac{(1+\lambda) \ln (1+\lambda)-\lambda}{\lambda}
\end{aligned}
$$

with $x \equiv \xi \gamma_{0} T(1-T) \Delta$ This is a supei position of binomial distributions The factorial cumulants are

$$
\left\langle\left\langle n^{k}\right\rangle\right\rangle_{\mathrm{f}}=(-1)^{k+1} \frac{(k-1)^{\prime}}{k+1} \frac{\tau \Delta}{2 \pi}\left[T(1-T) \gamma_{0} \Delta\right]^{k}
$$

The second factor ial cumulant is negative, so $\operatorname{Vat} n<\langle n\rangle$ This is sub-Poissoman tadiation

We have not found such a simple closed form expression in the more general multichannel case, but it is straightfor ward to evaluate the low-order factorial cumulants f1om Eq (10) We find

$$
\begin{gathered}
\langle n\rangle=\frac{\tau \Delta}{2 \pi} \gamma_{0} \Delta \frac{1}{2} S_{1}, \\
\left\langle\left\langle n^{2}\right\rangle\right\rangle_{1}=\frac{\tau \Delta}{2 \pi}\left(\gamma_{0} \Delta\right)^{2} \frac{1}{3}\left(S_{1}^{2}-2 S_{2}\right), \\
\left\langle\left\langle n^{3}\right\rangle\right\rangle_{\mathrm{f}}=\frac{\tau \Delta}{2 \pi}\left(\gamma_{0} \Delta\right)^{3} \frac{1}{6}\left(3 S_{1}^{3}-15 S_{1} S_{2}+15 S_{3}\right),
\end{gathered}
$$

with $S_{p}=\sum_{n}\left[T_{n}\left(1-T_{n}\right)\right]^{p} \quad$ Antibunching therefore 1equiles $S_{1}^{2}<2 S_{2}$

The condition on antibunching can be generalized to atbitiaty fiequency dependence of the response function $\gamma(\omega)$ in the range $V-\Delta<\omega<V$ of detected frequencies For $\Delta<V / 2$ we find

$$
\begin{aligned}
\text { Vain } n-\langle n\rangle= & \frac{\tau}{2 \pi} \int_{V-\Delta}^{V} d \omega^{\prime} \gamma\left(\omega^{\prime}\right) \int_{\omega^{\prime}}^{V} d \omega(V-\omega) \\
& \times\left[2 S_{1}^{2}-4 S_{2}-(V-\omega) S_{1}^{2} \frac{d}{d \omega}\right] \gamma(\omega)
\end{aligned}
$$

We see that the antibunching condition $S_{1}^{?}<2 S_{2}$ deilved for the special case of the block function (14) is more generally a sufficient condition for antibunching to occui, provided that $d \gamma / d \omega \geq 0$ in the detection range It does not matter if the response function diops off at $\omega>V$, 
provided that it increases monotonically in the range $(V-\Delta, V)$ A steeply increasing response function in this range is more favorable, but not by much For example, the power law $\gamma(\omega) \propto(\omega-V+\Delta)^{p}$ gives the antibunching condition $S_{1}^{2}<2 S_{2} \times[1+p /(1+p)]$, which is only weakly dependent on the power $p$

In conclusion, we have presented both a qualitative physical picture and a quantitative analysis for the convetsion of election to photon antibunching A simple criterion, Eq (18), is obtained for sub-Poissonian photon statistics, in teims of the transmission eigenvalues $T_{n}$ of the conductor Since an $N$-channel quantum point contact has only a single $T_{N}$ different fiom 0 or 1 , it should generate antibunched photons in a frequency band $(V-\Delta, V)$-regaidless of the value of $T_{N}$ The statistics of these photons is the supeiposition (15) of binomial distisutions, inherited fiom the electionic binomial distribution There are no stingent conditions on the band width $\Delta$, as long as it is $<V / 2$ (in order to prevent mult1photon excitations by a single electron [16]) This should make it feasible to use the cross-coirelation technique of Ref [1] to detect the emission of nonclassical microwaves by a quantum point contact

We have benefited fiom collespondence with D C Glattl 1 This work was suppoited by the Dutch Science Foundation NWO/FOM

[1] J Gabell1, L-H Reydellet, G Feve, J M Berron, B Plaçaıs, P Roche, and D C Glattlı, Phys Rev Letl 93, 056801 (2004)

[2] R Hanbui y Brown and R Q Twiss, Nature (London) 177, 27 (1956)

[3] C W J Beenakke and H Schomerus, Phys Rev Lett 86, $700(2001)$

[4] Sub-Poissonian radiation is called "nonclassical" because its photocount statistics cannot be interpreted in classical teims as a superposition of Poisson processes See L Mandel and E Wolf, Optical Coherence and Quantum Optrcs (Cambidgc Unıversity, Cambridge, 1995)
[5] J Kım, O Benson, H Kan, and Y Yamamoto, Nature (London) 397, 500 (1999), C Santor1, M Pelton, $G$ Solomon, Y Dale, and Y Yamamoto, Phys Rev Lett 86, 1502 (2001)

[6] P Michleı, A Imamoğlu, M D Mason, P J Caıson, G F Strouse, and S K Buratto, Nature (London) 406, 968 (2000), P Michler, A Kıraz, C Bechet, W V Schocnfeld, $\mathrm{P} M$ Petıoff, L Zhang, E Hu, and A Imamoğlu, Science 290, $2282(2000)$

[7] Z L Yuan, B E Kardynal, R M Stevenson, A J Shıelds, C J Lobo, K Cooper, N S Beattie, D A Ritchie, and M Pepper, Science 295, 102 (2002)

[8] L S Levitov and G B Lesovik, JETP Lell 58, 230 (1993)

[9] The negatlve-bunomial distubution $P(n) \circ(n+1-1) \times$ $[\nu /\langle n\rangle+1]^{-n}$ counts the number of partitions of $n$ bosons among $\nu=\tau \delta \omega / 2 \pi$ states in a fiequency interval $\delta \omega$ The binomal distiubution $P(n) \approx \nu n[\nu /\langle n\rangle-1] "$ counts the number of patitions of $n$ fermions among $\nu$ states

[10] Equation (6) is the multimatix generalization of the well-known identity $\exp \left(b^{\dagger} A b\right)=\mathcal{N} \exp \left[b^{\dagger}\left(e^{A}-1\right) b\right]$

[11] K E Cahıll and R J Glauber, Phys Rev A 59, 1538 (1999)

[12] R Aguado and L P Kouwenhoven, Phys Rev Lett 84, $1986(2000)$

[13] The saddle point is at $z_{p}=0$, so to integrate out the Gaussian fluctuations atound the saddlc point we may linearıze the determinant in Eq (10) $\prod_{n} \operatorname{Det}\left[1+T_{n}(1-\right.$ $\left.\left.T_{n}\right) \xi X\right]=\exp \left[\xi S \mathrm{Ti}_{1} X+\mathcal{O}\left(X^{2}\right)\right]$ The result is Eq (12)

[14] Factorial cumulants are constructed from factorial moments in the usual way The fist two are $\langle\langle n\rangle\rangle_{1}=\langle n\rangle$ $\left\langle\left\langle n^{2}\right\rangle\right\rangle_{1}=\left\langle n^{2}\right\rangle_{f}-\langle n\rangle^{2}=\mathrm{Val} n-\langle n\rangle$

[15] Using computer algebra, we find that $\ln \langle\operatorname{Det}[1+\xi T(1-$ $T) X]\rangle=\sum_{m-1}^{M} \ln \left[1+m \xi \gamma_{0} T(1-T)(2 \pi / \tau)\right]$, for each matilx dimensionality $M$ that we could check We are confident that this closed form holds for all $M$, but we have not yet found an analytical proof Equation (15) follows in the $\lim _{1 \mathrm{l}} M \equiv \tau \Delta / 2 \pi \rightarrow \infty$ upon conversion of the summation into an integration

[16] Multiphoton excitations do not contisute to Varn if $T_{n} \in\left\{\begin{array}{lll}0 & 1 / 2 & 1\end{array}\right\}$ for all $n$ [cf $\operatorname{Ref}$ [3], Eq (19)] Fol a quantum point contact, one finds that antibunching persists when $\Delta>V / 2$ piovided that $T_{N}\left(1-T_{N}\right)>1 / 6$ 